\title{
Onychogryphosis: a treatment with phenol derivatives and tocopherol instead of surgery, according to the ancient method employed in XIX century
}

\section{Piotr Brzeziński ${ }^{1}$, Lorenzo Martini ${ }^{1,2}$}

${ }^{1}$ University of Siena, Department of Pharmaceutical Biotechnologies, Via A. Moro 2, 53100 Siena, Italy, ${ }^{2}$ C.R.I.S.M.A. Inter

University Centre for Researched Advanced Medical Systems

Corresponding author: Prof. Lorenzo Martini, E-mail: martini36@unisi.it

Sir,

Onychogryphosis is a nail disease that causes one side of the nail to grow faster than the other. The nickname for this disease is ram's horn nails because the nails are thick and curvy, like horns or claws. Onychogryphosis mostly affects the toes - specifically the big toes. The morphology of injured nail seems an oyster shell.

In case of onychogryphosis, nails will look: yellow or brown; unusually thick; long (extending beyond the toe); curved

Ram's horn nails can develop at a variety of ages depending on the underlying cause. It can be especially problematic for young adults and older adults. In case of this syndrome it is necessary to seek a specific treatment. The condition will get worse with time, and can also cause: ingrown nails; pain; infection; inability to pursue physical activities like sports or physically active careers; time away from work.

The chief cause of onychogryphosis are six:

1. Foot trauma

Repeatedly hurting feet - or minor foot trauma - can damage the toes and nail plates, eventually leading to onychogryphosis. For example, wearing shoes that are too small for the person who wear it every day can cause foot trauma. Onychogryphosis can also develop if the patient has a condition like hammer toe. Treatment can be as simple as wearing shoes that are the correct size. It is possible also use splints and pads to train the toes and nails to grow normally.

2. Fungal infection

Onychomycosis is a fungal infection that causes the nails to become thick, crinkly, and brittle, albeit it can drive to onycogryphosis. This infection mostly affects toenails but can also impact fingernails.

Research shows up to 50 percent Trusted Source of Onychomycosis cases complicate or may lead to ram's horn nails. Doctors diagnose Onychomycosis by examining skin tissue that is swabbed or scraped from under an affected nail. Oral and topical antibiotics can be used to treat fungal infections.

3. Psoriasis

Psoriasis is a fairly common autoimmune disease that causes the body to produce extra skin cells. These extra cells build up and form red, dry, scaly patches of skin. These skin growths can also affect the nails. Roughly half of people with psoriasis experience nail changes. Roughly one third of people with nail psoriasis have onychomycosis.

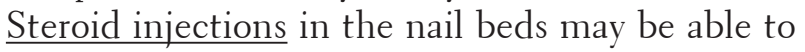
treat these growths. Taking antifungal medication may also help. If these treatments don't work, you may need surgery.

4. Peripheral vascular disease

Peripheral vascular disease, also called peripheral artery disease (PAD), causes the arteries in legs to build up with plaque. This reduces blood flow to legs and feet. Without proper blood flow, one may notice sores on his legs or feet, and slow or unusual nail growth. If left untreated, PAD can lead to onychogryphosis. Smoking is a main risk factor

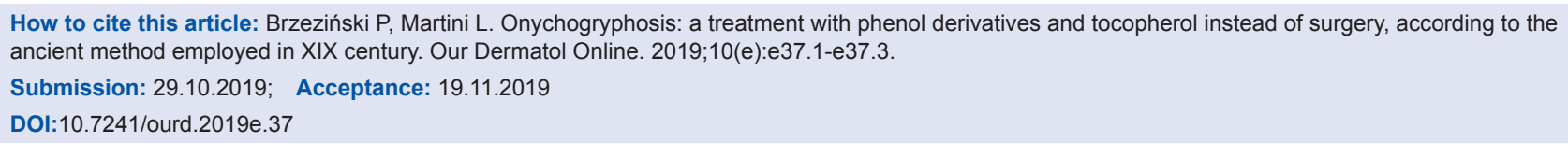


for developing PAD. Treatment options include lifestyle changes, medication, and surgery to clear the artery of plaque.

5. Ichthyosis

Ichthyosis is a rare skin condition that prohibits the body from shedding dead skin cells. A common symptom of this genetic condition is thickened or deformed nails, which can turn into onychogryphosis in some cases. Ichthyosis is normally diagnosed at birth when a baby is born with collodion membrane on their skin. Topical creams and oral retinoids are the most common treatment methods. If onychogryphosis develops, surgery may be necessary.

6. Tuberous sclerosis complex

Tuberous sclerosis complex (TSC) is a rare genetic disease that causes benign tumors to grow throughout the body. TSC is typically diagnosed because of the skin problems associated with it, including nail deformities. While the nail deformities go away in some cases, they may get worse over time, turning into ram's horn nails. Other symptoms of TSC include cognitive impairment, autism, and seizures. Treatment for ram's horn nails associated with TSC is surgery.

Surgery is generally the only accepted treatment option for onychogryphosis [1]. The type and frequency of the surgery, however, is based on the cause of ram's horn nails. If the condition is genetic, the patient may have to get the same surgery multiple times as the nails grow back. If the cause is less severe, such as foot trauma or infection, it is advisable to perform surgery to correct the problem. Nails should be clipped straight across rather than curved to avoid ingrown nails. The patient who has been cured should also wear clean cotton socks that can absorb moisture and help prevent fungal infections.

Additional treatment methods may be used to address the underlying cause of ram's horn nails to prevent the condition from developing [2].

The managing of ram's horn nails, keeping on account that they are also painful and can severely impact the quality of life [3].

While it's not always possible to prevent onychogryphosis, here follows a few simple saga cities and shrewd nesses a patient can do to promote nail health [4].

- keep nails trimmed short

(c) Our Dermatol Online e.2019
- cut nails so they are straight, rather than curved at the edges

- wear shoes that fit and have enough room in the toe box

- wear cotton socks that absorb moisture

- change socks regularly

- wear gloves when handling chemicals

Ram's horn nails can be also treated by:

- wearing adapted shoes

- regularly visiting a podiatrist

The AA have decided to revisit the ancient method of curing this disease.

In XIX century, when only wealthy person could afford hygienic accommodations, this nail disease was treated using phenol.

Now pure phenol was isolated in 1834 and its structure proved in 1842. In 1860 Küchenmeister first used phenol as a wound dressing in Germany [5].

Today phenol is not allowed as cosmetic ingredient but the AA have created a mix of phenol derivatives that are allowed in INCI and have treated a severe case of onychogryphosis, obtaining excellent results.

The AA have chosen a single case of a man (86 y. old) with a severe onychogryphosis (in the left big toe).

The nail was yellowish-greenish and horny.

The combination the AA have used was based on these five phenol derivatives, that are admitted in INCI.

(Dinonylphenol; 2,3 xylenol; Hydrogenated bisphenol; Peg 10 tris phenylethyl phenol; BHT, Tocopheryl acetate $[6,7])$.

The solvent was amylic alcohol and the cosmetic system was similar to a nail enameling and was applied thanks to a little brush on the big toe.

The treatment lasted 3 weeks, but the beginning of the remission was evident after the $5^{\text {th }}$ day.

The nail of big toe grows softer and softer during the first 5 days and after for 6 days the colour of the nail begins to assume a whitish and translucent.

After the $18^{\text {sth }}$ day the nail can be cut and modelled. 


\section{Consent}

The examination of the patient was conducted according to the Declaration of Helsinki principles.

\section{REFERENCES}

1. Ghaffarpour G, Azizian Z, Ghasemi MR. A new surgical technique in treatment of nail onycogryphosis. World J Plast Surg. 2018;7:136-8.

2. Chang P, Domínguez K. [Nail diseases in elderly. Report of 71 cases]. Our Dermatol Online. 2016;7:385-90.

3. Chang P. Onychogryphosis. Our Dermatol Online. 2011;2:227-8.

4. Abreu Velez AM, Upegui-Zapata YA, Valencia-Yepes CA, Upegui-
Quiceño E, Jiménez-Echavarria AM, Niño-Pulido CD, et al. Nail alterations in patients affected by endemic pemphigus foliaceus in el Bagre, Colombia. Our Dermatol Online. 2019;10:325-8.

5. Vermerris W, Nicholson R. Phenolic Compound Biochemistry Springer, 2008.

6. Fiegein H. "Cresols and Xylenols" in Ullmann's Encyclopedia of Industrial Chemistry, 2007; Wiley-VCH, Weinheim.

7. CRC Handbook of Tables for Organic Compound Identification, Third Edition, 1984.

Copyright by Piotr Brzeziński, et al. This is an open-access article distributed under the terms of the Creative Commons Attribution License, which permits unrestricted use, distribution, and reproduction in any medium, provided the original author and source are credited.

Source of Support: Nil, Conflict of Interest: None declared. 